\title{
Electrochemistry, morphology, thermoelectric and thermal degradation behaviors of free-standing copolymer films made from 1,12-bis(carbazolyl) dodecane and 3,4-ethylenedioxythiophene
}

\author{
Ruirui Yue, Baoyang Lu, Jingkun Xu, Shuai Chen and Congcong Liu
}

\begin{abstract}
A copolymer based on 1,12-bis(carbazolyl)dodecane (2Cz-D) and 3,4-ethylenedioxythiophene (EDOT) was electrochemically synthesized in dichloromethane containing 0.1-m tetrabutylammonium tetrafluoroborate. Cyclic voltammetry, Fourier-transform infrared, morphological and elemental analyses confirm that the resultant polymer is a copolymer rather than a composite or a blend of the two homopolymers. The copolymer exhibits good redox activity and high electrochemical stability. In contrast with powdered poly(3,4-ethylenedioxythiophene) (PEDOT), the copolymer formed in this study exists in a free-standing film state, and it has a higher thermal stability and better mechanical properties. Moreover, the copolymer films emit blue light, depending on the monomer feed ratios. The thermoelectric properties of the copolymer films were also investigated. Because the 2CZ-D/EDOT feed ratios were different, the electrical conductivities of the obtained copolymer films were improved to varying degrees compared with that of the poly(1,12-bis(carbazolyl)dodecane) (P2Cz-D) film. The highest obtained electrical conductivity was $0.6 \mathrm{~S} \mathrm{~cm}^{-1}$, four orders of magnitude higher than that of P2Cz-D $\left(2.7 \times 10^{-5} \mathrm{~S} \mathrm{~cm}^{-1}\right)$. The Seebeck coefficients of the copolymer films were also modified compared with that of P2Cz-D. All of these enhanced properties will be beneficial to the potential applications of these materials in polymer optoelectronics or as organic thermoelectric materials.
\end{abstract}

Polymer Journal (2011) 43, 531-539; doi:10.1038/pj.2011.26; published online 27 April 2011

Keywords: carbazole; conducting polymers; electrochemical copolymerization; 3,4-ethylenedioxythiophene; fluorescence; thermoelectric properties

\section{INTRODUCTION}

In spite of their short history, conducting polymers $(\mathrm{CPs})$ have attracted considerable attention as an emerging area of polymeric materials for applications in electronic and photonic devices by virtue of their metal-like electrical conductivity in conjunction with their polymeric properties such as flexibility, low density and ease of structural modification. ${ }^{1-6}$ However, despite a great deal of work focused on fundamental research on conventional $\mathrm{CPs}$, the design and synthesis of novel CPs with unique properties are still seen as necessary and significant to enable the application of these materials.

Carbazole-containing polymers have generated significant interest because of their applications in electrochromic devices, hole-transporting layers, electroxerography, microcavity photoconduction and as photovoltaic components that provide a highly efficient matrix as a current carrier transport. ${ }^{7-11}$ These polymers also constitute a significant portion of photoconductive polymers and organic photoreceptors..$^{12}$ Carbazole can be easily functionalized at its $3,6-,^{13,14} 2,7_{-}{ }^{15}$ or $\mathrm{N}-16,17$ positions and then covalently linked to a polymer, either in the main chain as a block ${ }^{18}$ or in the side chain as a pendant group. ${ }^{19}$
Dicarbazoles connected with various etheroxide spacers or carbon chains have been investigated. ${ }^{20-24}$ The increasing polymerization positions, together with the flexible spacers between the chromophores, lead to the crosslinked polymer structures. Owing to the ease of formation of relatively stable radical cations (polarons), carbazoles readily polymerize in electrochemical polymerization processes. As reported by our group, poly(1,12-bis(carbazolyl)dodecane) (P2Cz-D) free-standing films with high mechanical properties and outstanding blue-light emitting properties can be easily obtained through direct anodic oxidation of 1,12-bis(carbazolyl)dodecane (2Cz-D) in a neutral system. ${ }^{25}$ However, the as-formed P2Cz-D films show relatively poor conductivity, which impedes their practical applications.

Generally, the properties of CPs can be tuned by adjusting the electronic characteristics of the $\pi$-orbital along the neutral polymer backbone, including main-chain and pendant-group structural modification and copolymerization. ${ }^{26,27}$ As a general synthetic method, copolymerization is a promising strategy that can result in the combination of the properties of the homopolymers. For that matter, many recent studies have reported novel copolymers based on

College of Chemistry and Chemical Engineering, Jiangxi Key Laboratory of Organic Chemistry, Jiangxi Science and Technology Normal University, Nanchang, China Correspondence: Professor J Xu, Jiangxi Key Laboratory of Organic Chemistry, Jiangxi Science and Technology Normal University, Fenglin Street, Nanchang 330013, China. E-mails: xujingkun@tsinghua.org.cn or xujingkun@mail.ipc.ac.cn

Received 27 November 2010; revised 21 February 2011; accepted 22 February 2011; published online 27 April 2011 
3,4-ethylenedioxythiophene (EDOT) and other components. ${ }^{28-31}$ EDOT is a popular choice as a comonomer because it produces a low band gap polymer with high stability and good conductivity. ${ }^{32}$ EDOT and its derivatives can give rise to non-covalent intramolecular interactions with adjacent thiophenic units, thus inducing the selfrigidification of the conjugated system in which it is incorporated. ${ }^{33,34}$

On the basis of the above considerations, the electrochemical copolymerization of $2 \mathrm{Cz}-\mathrm{D}$ and EDOT was carried out in $\mathrm{CH}_{2} \mathrm{Cl}_{2}$ containing $\mathrm{Bu}_{4} \mathrm{NBF}_{4}$ as the supporting electrolyte. The resultant copolymer was characterized by means of Fourier-transform infrared (FT-IR), elemental analysis, scanning electron microscope and cyclic voltammetry. The light-emitting properties, thermoelectric behavior and thermal stability of the copolymer films were studied in detail.

\section{EXPERIMENTAL PROCEDURE}

\section{Materials}

2Cz-D was synthesized according to general procedures already described in the literature. ${ }^{35}$ 3,4-Ethylenedioxythiophene (EDOT, Sigma-Aldrich, Shanghai, China, 98\%) and 1,12-bisbromo-dodecane (Acros, Shanghai, China) were used as received. Tetrabutylammonium tetrafluoroborate $\left(\mathrm{Bu}_{4} \mathrm{NBF}_{4}\right.$, Acros) was dried at $60^{\circ} \mathrm{C}$ for $12 \mathrm{~h}$ before use. $\mathrm{CH}_{2} \mathrm{Cl}_{2}$ (AR, Beijing Chemical Plant, Beijing, China) was refluxed with $\mathrm{CaH}_{2}$ and distilled before use. All other reagents are commercial-grade products and were used as received. Indium-tin-oxide (ITO)coated glass (Shenzhen Changda Electronic Company, Shenzhen, China) was washed with ethanol and then with deionized water under ultrasonication and was further dried in air.

\section{Electrochemical synthesis and testing}

Electrochemical polymerization and examination were carried out in a onecompartment three-electrode cell with a Model 263 potentiostat-galvanostat (EG\&G Princeton Applied Research, Beijing, China) under computer control at the temperature of ice water. The working and counter electrodes in cyclic voltammetry experiments were two platinum wires placed $0.5 \mathrm{~cm}$ apart, both with $0.5-\mathrm{mm}$ diameters. Before each examination, the wires were carefully polished and cleaned with water and then with acetone. $\mathrm{An} \mathrm{Ag} / \mathrm{AgCl}$ electrode immersed directly in the solution was used as the reference electrode. All solutions were deaerated with a dry argon stream before experiments. To obtain amounts of polymer films, a platinum sheet with a surface area of $4.5 \mathrm{~cm}^{2}$ and a stainless steel sheet with a surface area of $5.0 \mathrm{~cm}^{2}$ were used as the working and counter electrodes, respectively. These sheets were carefully polished with abrasive paper (1500 mesh) and then washed with water and then with acetone before each examination. The polymers deposited on ITO glass (about $2.0 \mathrm{~cm}^{2}$ ) were used for ultraviolet-vis, fluorescence and scanning electron microscope measurements. For specific analysis, the as-formed polymers were dedoped with $25 \%$ ammonium for 2 days and then washed repeatedly with water and acetone. Finally, the samples were dried at $60^{\circ} \mathrm{C}$ for 2 days under vacuum.

\section{Characterization}

The temperature dependence of the electrical conductivity $(\sigma)$ and the Seebeck coefficient $(S)$ were measured by a four-point thermoelectric property measurement unit coupled with a liquid-nitrogen container for rectangular-shaped film (length: $12.0-13.0 \mathrm{~mm}$, width: $3.0-3.5 \mathrm{~mm}$, thickness: $0.03-0.10 \mathrm{~mm}$ ) or pellet (length: $15.0 \mathrm{~mm}$, width: $3.0 \mathrm{~mm}$, thickness: $1.0-2.0 \mathrm{~mm}$ ) samples from room temperature to $200 \mathrm{~K}$. The Seebeck coefficient $(S)$ was obtained from the slope of the produced thermoelectric voltage as a function of the temperature difference along the length of the sample.

Ultraviolet-vis spectra were measured with a Perkin-Elmer Lambda 900 UV-visible-near-infrared spectrophotometer (Perkin-Elmer, Beijing, China). Fluorescence spectra were captured using an F-4500 fluorescence spectrophotometer (Hitachi, Beijing, China). Infrared spectra were recorded with a Bruker Vertex 70 Fourier-transform infrared spectrometer (Bruker, Beijing, China) with samples in $\mathrm{KBr}$ pellets. Mechanical properties were measured by an Instron 2365 universal testing instrument (Instron, Shanghai, China) at a stretching rate of $5 \mathrm{~mm}$ per minute. Elemental analyses were performed with an S-4700 X-ray elemental analyzer (Hitachi). A VEGA|LSU Tescan scanning electron microscope (Tescan, Shanghai, China) was used to analyze the surface morphologies of as-formed polymer films. The thermal stability of the polymers was analyzed by a Pyris Diamond TG/DTG thermal analyzer (Perkin-Elmer) under a nitrogen stream with a heating rate of $10 \mathrm{~K} \mathrm{~min}^{-1}$.

\section{RESULTS AND DISCUSSION}

\section{Electrochemical polymerization}

Cyclic voltammetry is a highly successful method that qualitatively reveals the reversibility of electron transfer during electrochemical polymerization and also examines the electrical activity of polymer films by monitoring the oxidation and reduction in the form of a current-potential diagram (cyclic voltammogram, CV). ${ }^{36}$ To investigate the electrochemical polymerization behavior of the monomer mixtures and the effect of the initial monomer feed ratio on the copolymerization process, CVs of $2 \mathrm{Cz}-\mathrm{D}$, EDOT and that of the monomer mixtures with different feed ratios were recorded and are shown in Figure 1 and Supplementary Figure S1. The growth of the $\mathrm{CPs} /$ copolymers appears as an increasing redox peak current density on each CV. Figure 1a displays the CV of $2 \mathrm{Cz}$-D performed between 1.27 and $0.3 \mathrm{~V}$. The onset oxidation potential of $2 \mathrm{Cz}-\mathrm{D}$ is $1.10 \mathrm{~V}$ lower than that of EDOT $(1.33 \mathrm{~V})$ shown in Figure 1c, observed from the first circle of the CVs. The small difference in the oxidation potential between $2 \mathrm{Cz}-\mathrm{D}$ and EDOT $(0.23 \mathrm{~V})$ suggests that copolymerization between the two is possible. Upon repeated scanning, a reversible redox process develops at 0.85 and $0.70 \mathrm{~V}$ for $2 \mathrm{Cz}-\mathrm{D}$ (Figure 1a), suggesting the formation of an electroactive polymer with good redox reversibility. For the EDOT CVs, typical broad redox peaks can be observed between 1.2 and $0.3 \mathrm{~V}$ (Figure 1c). Figure $1 \mathrm{~b}$ presents the $\mathrm{CVs}$ of the monomer mixtures with $2 \mathrm{Cz}-\mathrm{D}: \mathrm{EDOT}=1: 5$. Similar to the $\mathrm{CV}$ of $2 \mathrm{Cz}-\mathrm{D}$ (Figure 1a), the mixture displays redox peaks at 1.0 and $0.6 \mathrm{~V}$, which are much broader than those of $2 \mathrm{Cz}-\mathrm{D}$ but are consistent

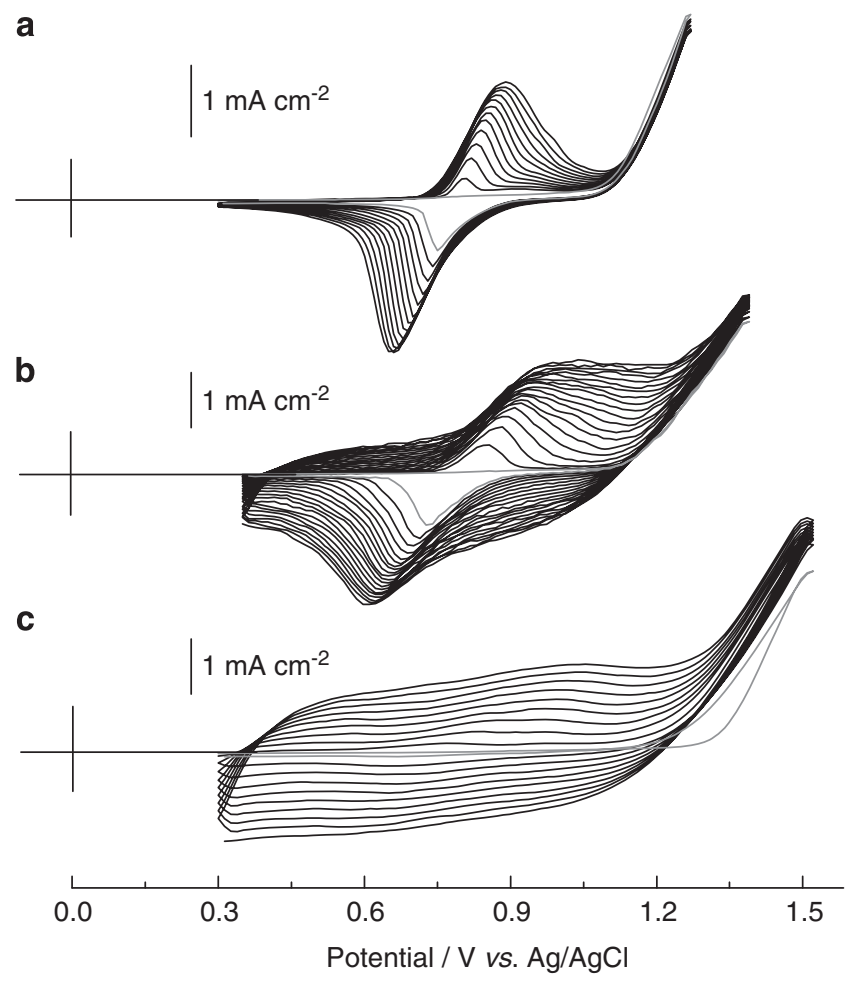

Figure 1 Cyclic voltammograms of 2Cz-D (a), EDOT (c) and monomer mixtures with a feed ratio of $2 \mathrm{Cz}-\mathrm{D}$ :EDOT $=1: 5$ (b) in $\mathrm{CH}_{2} \mathrm{Cl}_{2}+0.1-\mathrm{M}$ $\mathrm{Bu}_{4} \mathrm{NBF}_{4}$. Potential scan rate: $100 \mathrm{mVs}^{-1}$. 
with those of EDOT (Figure 1c). As the CV scanning continued, the redox waves underwent a clear shift in potential, indicating the increase in the electrical resistance of the as-formed polymer film and thus the overpotential needed to overcome the resistance. ${ }^{37}$ Similar phenomena can also be observed in Supplementary Figure $\mathrm{S} 1$. As the initial monomer feed ratio (2Cz-D:EDOT) decreased from 5:1 (Supplementary Figure S1a) to 1:10 (Supplementary Figure S1f), the CVs of the monomer mixtures changed gradually and became increasingly similar to that of EDOT (Figure 1c). The appearance of these new redox peaks confirms that some reactions must occur between 2Cz-D and EDOT during the electrochemical polymerization process in $\mathrm{CH}_{2} \mathrm{Cl}_{2}+0.1-\mathrm{M} \mathrm{Bu}_{4} \mathrm{NBF}_{4}$.

A high oxidation potential is known to make the electropolymerization of an aromatic monomer quite difficult and also to lead to some side reactions. Furthermore, the polymer films deposited on the working electrode surface are easily overoxidized during the further polymerization process, which takes place under a higher oxidation potential. Therefore, the preparation of high-quality conductive polymer films requires an appropriate polymerization potential. To choose an optimal polymerization potential for the monomer or monomer mixtures, a set of current transients were recorded during the potentiostatic polymerization and copolymerization processes at different applied potentials, as shown in Supplementary Figures S2 and S3. Considering the overall factors affecting the quality of the as-formed polymer films such as polymerization rate, unavoidable overoxidation, room temperature and adherence to the electrode surface, the applied polymerization potentials were chosen as $1.3 \mathrm{~V}$ for $2 \mathrm{Cz}-\mathrm{D}$ and $1.5 \mathrm{~V}$ for both EDOT and the monomer mixtures with different feed ratios in $\mathrm{CH}_{2} \mathrm{Cl}_{2}+0.1-\mathrm{M} \mathrm{Bu}_{4} \mathrm{NBF}_{4}$.

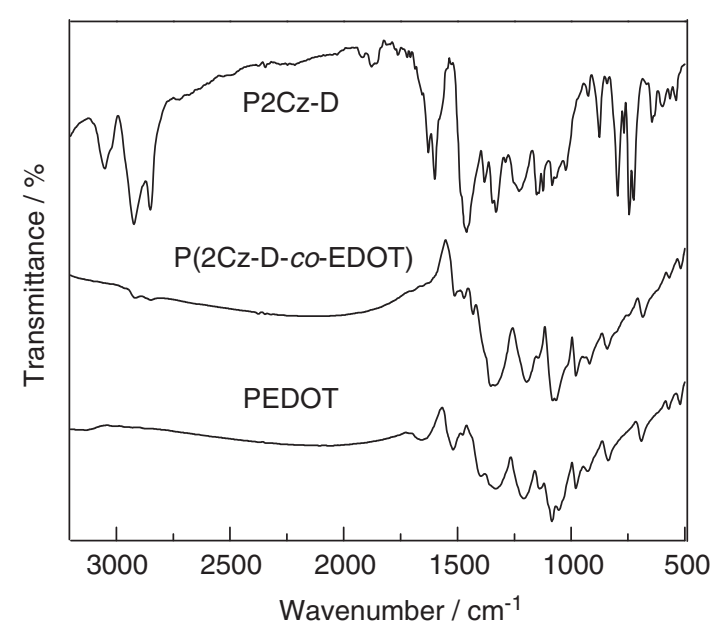

Figure 2 Fourier-transform infrared spectra of P2Cz-D, PEDOT and P(2Cz-Dco-EDOT) (2Cz-D:EDOT $=1: 5)$ in the dedoped state deposited from $\mathrm{CH}_{2} \mathrm{Cl}_{2}+0.1-\mathrm{M} \mathrm{Bu} \mathrm{NBF}_{4}$.

\section{Structural and morphological characterization}

Figure 2 shows the FT-IR spectra of P2Cz-D, poly(3,4-ethylenedioxythiophene) (PEDOT) and poly(2Cz-D/EDOT) obtained with $2 \mathrm{Cz}-$ $\mathrm{D}: \mathrm{EDOT}=1: 5$ in the dedoped state. According to the spectrum of $\mathrm{P} 2 \mathrm{Cz}-\mathrm{D}$, the band at $3058 \mathrm{~cm}^{-1}$ can be ascribed to the $\mathrm{C}-\mathrm{H}$ stretching vibration of benzene rings, and the bands at 2924 and $2840 \mathrm{~cm}^{-1}$ are assigned to the aliphatic $\mathrm{C}-\mathrm{H}$ stretching vibration. The band at $745 \mathrm{~cm}^{-1}$ suggests the existence of a 1,2-disubstituted benzene ring. The band at $712 \mathrm{~cm}^{-1}$ is ascribed to an out-of-plane deformation vibration of the long alkyl chain. ${ }^{25}$ In the PEDOT spectrum, the bands at 1519,1334 and $1208 \mathrm{~cm}^{-1}$ originate from the stretching modes of $\mathrm{C}=\mathrm{C}$ and $\mathrm{C}-\mathrm{C}$ in the thiophene rings, and the bands at 1090 and $972 \mathrm{~cm}^{-1}$ stem from the stretching vibrations of C-O-C and C-S-C in the EDOT rings, respectively. ${ }^{31}$ Compared with the constituent homopolymers, poly(2Cz-D/EDOT) exhibits bands at 2924, 2840 and $743 \mathrm{~cm}^{-1}$, indicating the presence of 2Cz-D units. The bands at 1343, 1191, 1073 and $981 \mathrm{~cm}^{-1}$ assigned to EDOT rings can also be observed. The above results demonstrate that poly (2Cz-D/EDOT) contains both 2Cz-D and EDOT units. Furthermore, according to previous studies, the electrochemical polymerization of carbazole and its derivatives mainly occurred at the 3,6 positions, ${ }^{38}$ suggesting that the copolymerization of $2 \mathrm{Cz}-\mathrm{D}$ with EDOT still occurred at the 3,6 positions.

As-formed P2Cz-D, PEDOT and poly(2Cz-D/EDOT) were all in the doped state and appeared black in color on platinum sheets. After dedoping with $25 \%$ ammonia, the color of the P2Cz-D film changed to brown, but PEDOT and poly(2Cz-D/EDOT) were still black, as shown in Figure 3, indicating the presence of PEDOT segments in poly $(2 \mathrm{Cz}-\mathrm{D} / \mathrm{EDOT})$ chains. However, as-formed P2Cz-D can be easily peeled off the electrode surface into a free-standing film with a smooth surface (Figure 3a). PEDOT polymerized under the same conditions as $\mathrm{P} 2 \mathrm{Cz}-\mathrm{D}$ appeared as a black powder (Figure 3c). As expected, the poly(2Cz-D/EDOT) films obtained by the copolymerization of $2 \mathrm{Cz}-\mathrm{D}$ and EDOT were free-standing with smooth and compact surfaces (Figure $3 \mathrm{~b})$. These results indicate that poly(2Cz-D/EDOT) is a copolymer rather than a composite or blend of $\mathrm{P} 2 \mathrm{Cz}-\mathrm{D}$ and PEDOT. Furthermore, the mechanical properties of the as-formed, free-standing copolymer films were studied, and their tensile strengths were measured as $125 \sim 90 \mathrm{~kg} \mathrm{~cm}^{-2}$, which was lower than that of the P2Cz-D film $\left(172 \mathrm{~kg} \mathrm{~cm}^{-2}\right)$. The measured tensile strength of poly(1,12-bis(carbazolyl)dodecane-co-3,4-ethylenedioxythiophene) $(\mathrm{P}(2 \mathrm{Cz}-\mathrm{D}-\mathrm{co}$-EDOT $))$ films depends on the initial monomer feed ratios. In general, increasing proportions of EDOT in the copolymer chain led to poorer mechanical properties of the copolymer films. The above data reveal that the as-formed copolymer films exhibit good mechanical properties, indicating the formation of crosslinks between conjugated chains with long alkyl chains as the bridge, as shown in Scheme 1.

Figure 4 shows the scanning electron microscopy images of P2Cz-D (a), PEDOT (b) and the copolymer (c) films deposited on ITO

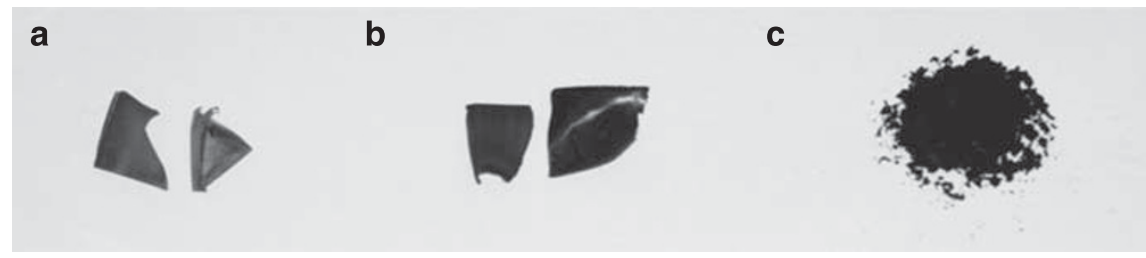

Figure 3 Photographs of as-formed free-standing P2Cz-D (a) and P(2Cz-D-co-EDOT) (2Cz-D:EDOT=1:5) (b) films and PEDOT powder (c) in the dedoped state electrosynthesized from $\mathrm{CH}_{2} \mathrm{Cl}_{2}+0.1-\mathrm{M} \mathrm{Bu}_{4} \mathrm{NBF}_{4}$. 

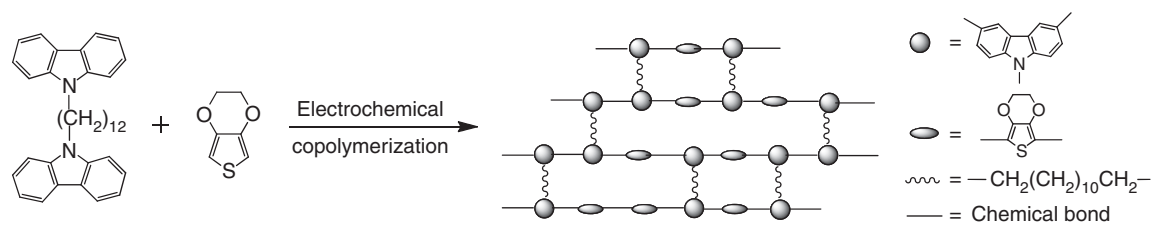

Scheme 1 Electrochemical copolymerization route of 2Cz-D and EDOT.
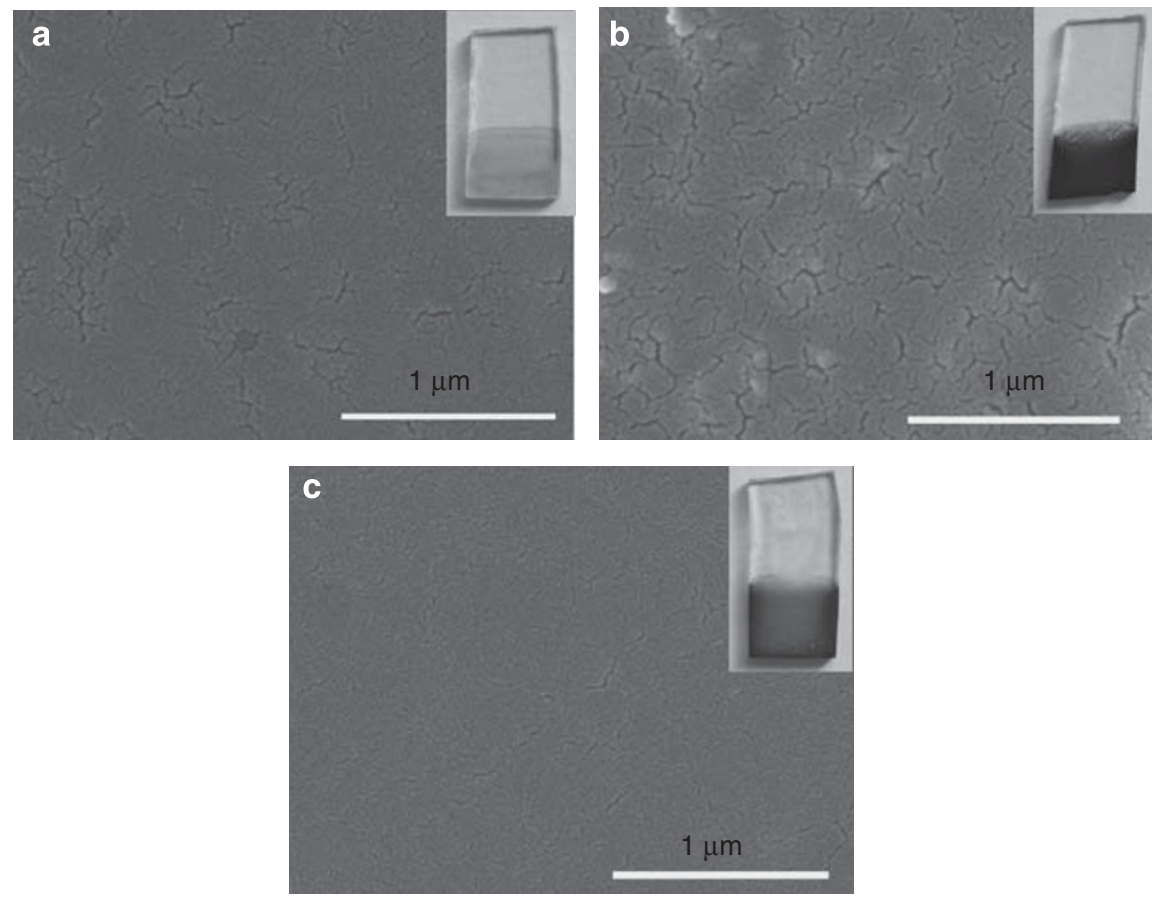

Figure 4 Scanning electron microscope images of P2Cz-D (a), PEDOT (b) and P(2Cz-D-co-EDOT) (2Cz-D:EDOT=1:5) (c) films synthesized in $\mathrm{CH}_{2} \mathrm{Cl}_{2}+0.1-\mathrm{M} \mathrm{Bu}_{4} \mathrm{NBF}_{4}$ at a constant applied potential of $1.30,1.50$ and $1.50 \mathrm{~V}$ for $100 \mathrm{~s}$, respectively. Insets: photographs of the corresponding polymer films deposited on indium-tin-oxide glasses in the doped state.

electrodes in the doped state (insets of Figure 4). Macroscopically, all the polymer films appeared to be smooth, homogeneous and compact. Microscopically, some crevices can be observed on the relatively smooth surface of the P2Cz-D film (Figure 4a). In contrast, the surface of the PEDOT film (Figure $4 \mathrm{~b}$ ) appears somewhat similar to dried and cracked earth, which could be partly attributed to the quick volatilization of $\mathrm{CH}_{2} \mathrm{Cl}_{2}$ from the polymer or the poor polymer film quality. By comparison, the as-formed copolymer film (Figure 4c) has a much smoother, more compact and more homogeneous surface with few tiny flaws, indicating the excellent crosslinking structure of the copolymer. In addition, the smooth and homogeneous structures of the compact copolymer films have an extremely beneficial effect on their electrical conductivity and also make them good candidates for applications such as ion-selective electrodes, ion-sieving films and matrices for hosting catalyst particles. ${ }^{39,40}$

To further explore the structure of the copolymer films obtained potentiostatically with different $2 \mathrm{Cz}-\mathrm{D} / \mathrm{EDOT}$ initial feed ratios, elemental analysis was performed and the results are shown in Table 1. Obviously, the ratio of $2 \mathrm{Cz}$-D/EDOT units in the corresponding copolymers is not proportional to the $2 \mathrm{Cz}$-D/EDOT feed ratio in $\mathrm{CH}_{2} \mathrm{Cl}_{2}+0.1-\mathrm{M} \mathrm{Bu} \mathrm{NBF}_{4}$, although the ratio of $2 \mathrm{Cz}-\mathrm{D} / \mathrm{EDOT}$ units in the copolymers decreases as the feed ratio decreases in $\mathrm{CH}_{2} \mathrm{Cl}_{2}$. When the feed ratio is $5: 1$, the $2 \mathrm{Cz}-\mathrm{D} / \mathrm{EDOT}$ ratio in the copolymer is 0.35 .
Table 1 Element analysis results of polymer films obtained with different $2 \mathrm{Cz}-\mathrm{D} / \mathrm{EDOT}$ ratios at constant applied potential of $1.5 \mathrm{~V}$ in $\mathrm{CH}_{2} \mathrm{Cl}_{2}+0.1 \mathrm{~m} \mathrm{Bu} \mathrm{NBF}_{4}$

\begin{tabular}{|c|c|c|c|c|c|}
\hline $\begin{array}{l}2 C Z-D / E D O T \\
\text { feed ratio }\end{array}$ & $C(\%)$ & $N(\%)$ & $O(\%)$ & $S(\%)$ & $\begin{array}{l}2 C z-D / E D O T \\
\text { ratio in polymer }\end{array}$ \\
\hline $5: 1$ & 83.2 & 1.07 & 4.09 & 1.53 & 0.35 \\
\hline $1: 1$ & 80.31 & 1.70 & 7.50 & 4.56 & 0.19 \\
\hline $1: 5$ & 81.55 & 1.78 & 8.88 & 5.23 & 0.17 \\
\hline $1: 10$ & 81.10 & 1.05 & 9.48 & 5.76 & 0.09 \\
\hline
\end{tabular}

Abbreviations: 2Cz-D, 1,12-bis(carbazolyl)dodecane; EDOT, 3,4-ethylenedioxythiophene.

When the feed ratio decreases to $1: 10$, the $2 \mathrm{Cz}-\mathrm{D} / \mathrm{EDOT}$ ratio in the copolymer decreases to 0.09 . Therefore, in the copolymer, P2Cz-D segments can be seen as the skeleton of the crosslinking structure, consistent with Scheme 1.

\section{Electrochemistry of copolymer films}

To obtain deeper insight into the electrochemical behavior of asformed copolymer films, the CVs of P(2Cz-D-co-EDOT) films deposited with different monomer feed ratios were recorded under 

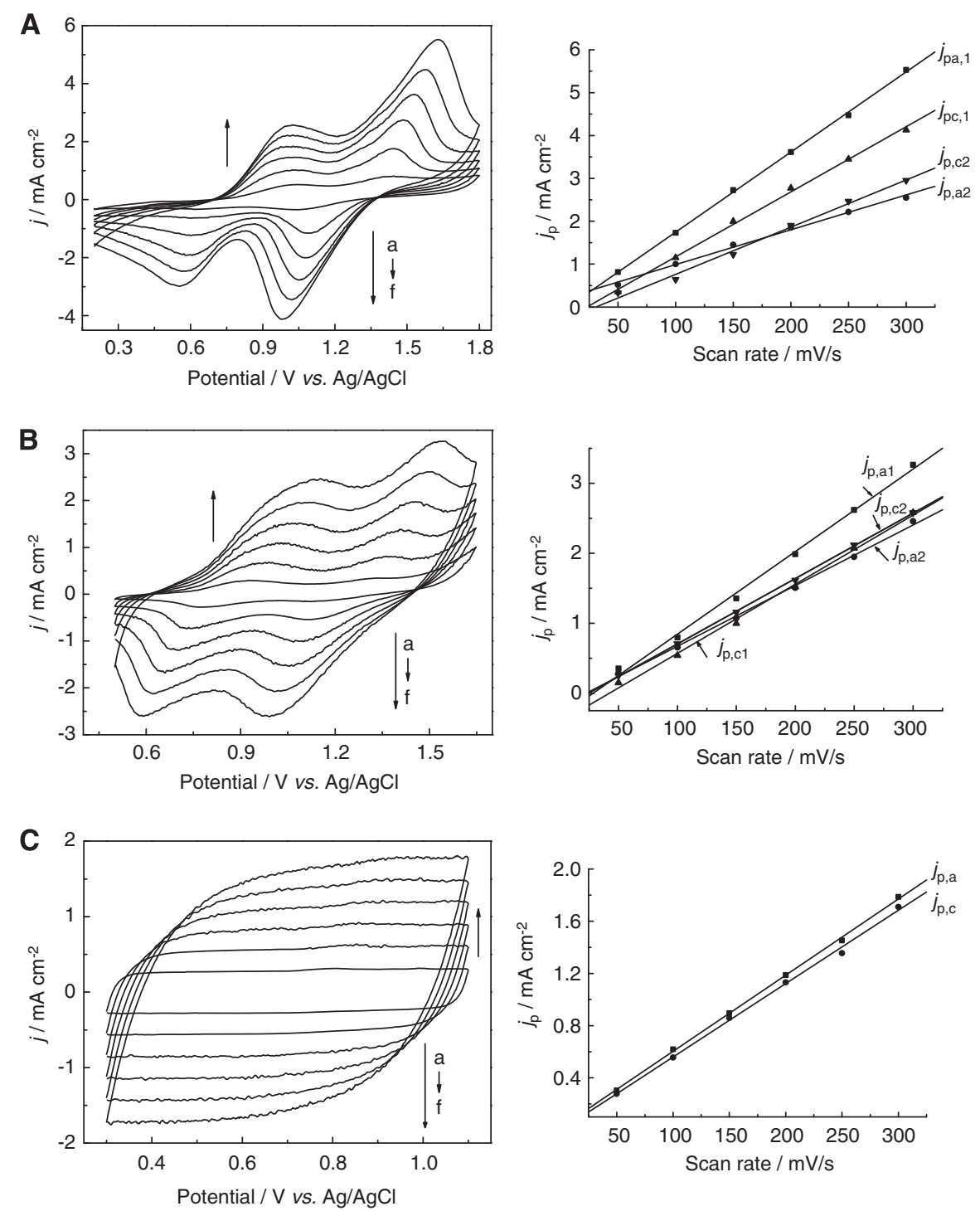

Figure 5 Cyclic voltammograms of P2Cz-D (A), P(2Cz-D-co-EDOT) (2Cz-D:EDOT=1:5) (B) and PEDOT (C) films recorded in monomer-free bulk solution at potential scan rates of 50 (a), 100 (b), 150 (c), 200 (d), 250 (e) and 300 (f) $\mathrm{mV} \mathrm{s}^{-1}$. The right plots show redox peak current densities vs potential scan rates. $j$ is peak current density, and $j_{\text {p.a. }}$ and $j_{\text {p.c. }}$. denote the anodic and cathodic peak current densities, respectively.

different potential scanning rates, as shown in Figure 5 and Supplementary Figure S4. For comparison, the CVs of P2Cz-D and PEDOT were also included in Figure 5. As shown in Figure 5A, the CVs of P2Cz-D show two pairs of redox peaks at 1.6 (anodic peak potential, $\left.E_{\text {p.a. }}\right)$ and $1.1 \mathrm{~V}$ (cathodic peak potential, $\left.E_{\text {p.c. }}\right)$, and at $1.0\left(E_{\text {p.a. }}\right)$ and $0.6 \mathrm{~V}\left(E_{\text {p.c. }}\right)$. For PEDOT, broad redox peaks can be found over the whole potential range as shown in Figure $5 \mathrm{C}$. Obviously, the CVs of the copolymer film (Figure 5B) displayed two pairs of broad redox peaks at $1.5\left(E_{\text {p.a. }}\right)$ and $1.0 \mathrm{~V}\left(E_{\text {p.c. }}\right)$, and at $1.1\left(E_{\text {p.a. }}\right)$ and $0.6 \mathrm{~V}\left(E_{\text {p.c. }}\right)$, similar to those of both $\mathrm{P} 2 \mathrm{Cz}-\mathrm{D}$ and PEDOT, and further indicating the presence of $2 \mathrm{Cz}-\mathrm{D}$ and EDOT units in the copolymer-conjugated backbone. Moreover, all the peak current densities were proportional to the potential scan rates (right parts of Figure 5), which suggests that the electroactive polymer films were well adhered and the redox processes were not diffusion limited. ${ }^{41}$ As the CVs were performed, these films could be cycled repeatedly between the conducting (oxidized) and insulating (neutral) states without significant material decomposition, indicating that these polymer have high redox stability. Similar behavior can also be observed in the CVs of other copolymer films synthesized with different monomer feed ratios (Supplementary Figure S4). As more EDOT was added, the CVs of the obtained copolymer films became increasingly similar to that of PEDOT, signifying that more EDOT units are present in the copolymer backbone chains. The above results further imply that the electrochemical copolymerization between 2Cz-D and EDOT in $\mathrm{CH}_{2} \mathrm{Cl}_{2}+0.1-\mathrm{M} \mathrm{Bu}_{4} \mathrm{NBF}_{4}$ occurred exactly as displayed in Scheme 1.

\section{Optical properties}

Thin P2Cz-D, PEDOT and copolymer films deposited on ITO glass are green, dark blue and cyan in color in the doped state, respectively, as shown in the insets of Figure 4 . When the same materials were dedoped at a negative potential, their colors changed to transparent colorless, dark brown and taupe, respectively. As-formed P2Cz-D, PEDOT and copolymers are all hardly soluble in common organic 
solvents such as DMSO, chloroform, tetrahydrofuran, dichloromethane and acetonitrile, which might result from the long conjugated backbone chains and their special crosslinking structure.

Figure 6A shows the ultraviolet-vis spectra of P2Cz-D (a), PEDOT (e) and copolymer films (b-d) in the doped state on ITO electrodes. For P2Cz-D (a), the spectrum displays absorption peaks at 400 and $825 \mathrm{~nm}$. Similarly, the copolymers show absorption peaks at $395 \mathrm{~nm}$ caused by the valence band-conduction band $\left(\pi-\pi^{*}\right)$ transition, and wide absorption bands centered at $841 \mathrm{~nm}$, indicating the presence of conductive species such as polaron or bipolaron and the long conjugated chain (b-d). However, as Figure 6Ae shows, PEDOT exhibits a much wider absorption band from 480 to more than $1000 \mathrm{~nm}$, and this broad peak is characteristic of the absorptions of conductive species. All the above features suggest that polymers with a long conjugated structure and a high electroactivity have been formed.

P2Cz-D is a good blue-light emitter. Thus, the fluorescence properties of the copolymers were investigated and are presented in Figure 6B. For comparison, the fluorescence spectra of P2Cz-D and PEDOT are also included. In the spectrum of $\mathrm{P} 2 \mathrm{Cz}-\mathrm{D}$ (a), an emission
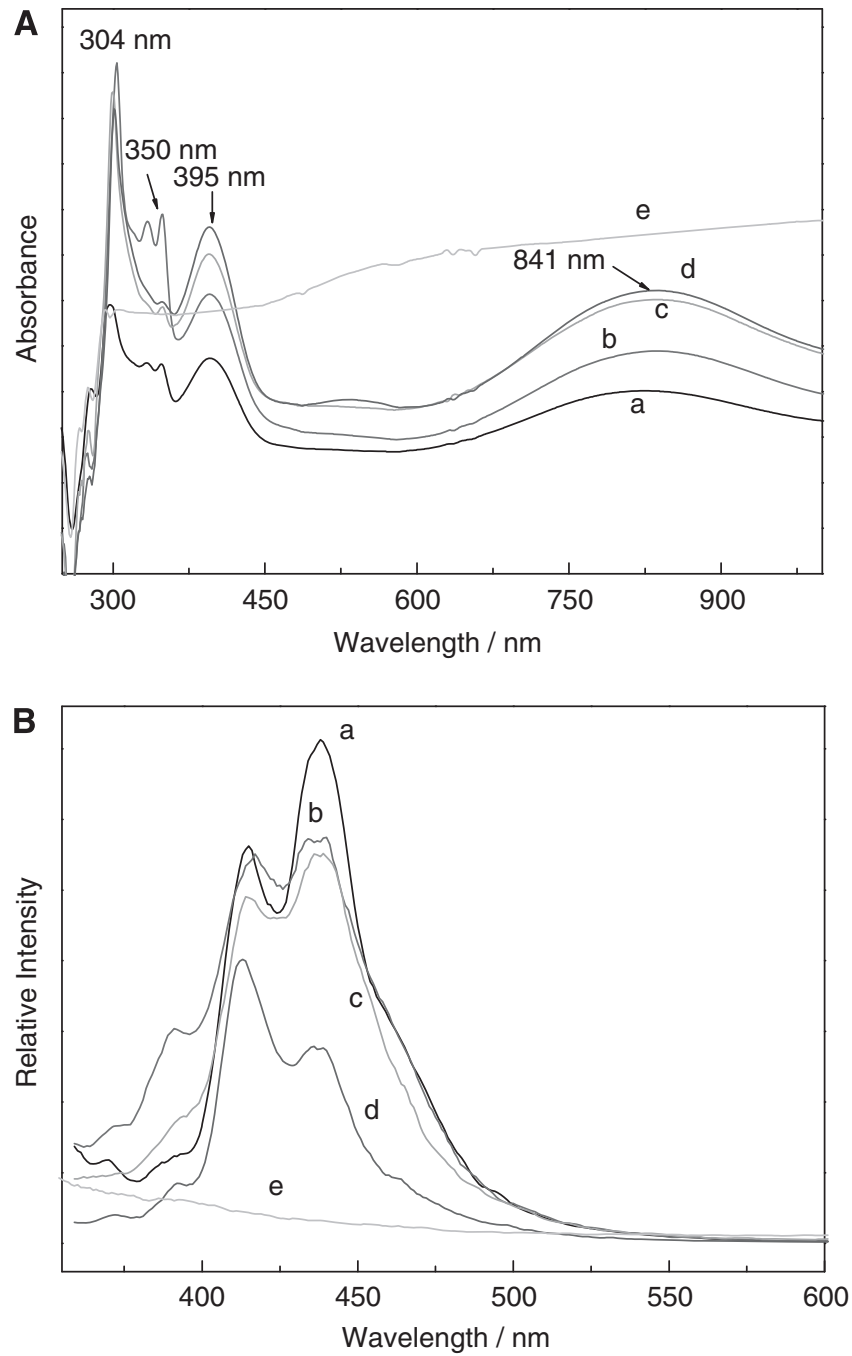

Figure 6 Ultraviolet-vis spectra (A) and emission spectra (B) of doped P2Cz-D (a) copolymers deposited with 2Cz-D:EDOT=5:1 (b), 1:1 (c) and $1: 10$ (d) and PEDOT (e) films coated on indium-tin-oxide glasses from $\mathrm{CH}_{2} \mathrm{Cl}_{2}+0.1-\mathrm{M} \mathrm{Bu}_{4} \mathrm{NBF}_{4}$. peak at $438 \mathrm{~nm}$ with a shoulder peak at $414 \mathrm{~nm}$ is observed. For the copolymers synthesized with $2 \mathrm{Cz}-\mathrm{D}: \mathrm{EDOT}=5: 1$ and $1: 1$ (b and $\mathrm{c}$ ), their emission spectra exhibit similar peaks with that of P2Cz-D. An emission peak at $436 \mathrm{~nm}$ with a shoulder at $415 \mathrm{~nm}$ can be found, indicating that the insertion of EDOT units into the polymer chain has not destroyed the original conjugated structure of P2Cz-D segments but rather filled in the gaps between adjacent $\mathrm{P} 2 \mathrm{Cz}$-D segments. When the $2 \mathrm{Cz}-\mathrm{D} / \mathrm{EDOT}$ feed ratio decreased to 1:10 (d), the emission peak of the as-obtained copolymer is located at $413 \mathrm{~nm}$ with a shoulder at $437 \mathrm{~nm}$, suggesting that more EDOT units inserted into the polymer chain and a slight fluorescence quenching appeared. As shown in Figure 6Be, PEDOT has no emission peaks in the whole visible region. However, its poor emission properties have little effect on those of the copolymers. Thus, similarl to $\mathrm{P} 2 \mathrm{Cz}-\mathrm{D}$, these obtained copolymers are still good blue-light emitters.

\section{Thermoelectric properties}

Figure 7 shows the temperature dependence of the electrical conductivities of P2Cz-D, PEDOT and the copolymers. The copolymer films were deposited potentiostatically with different 2Cz-D/EDOT feed ratios at the same applied potential of 1.5 V. As shown in Figure 7, all the electrical conductivities of the polymers gradually decreased as the temperature decreased from 300 to $200 \mathrm{~K}$, typical for semiconducting materials. ${ }^{42,43}$ The P2Cz-D film had an electrical conductivity at ambient temperature of $2.7 \times 10^{-5} \mathrm{~S} \mathrm{~cm}^{-1}$, and it gradually decreased to $2.3 \times 10^{-5} \mathrm{~S} \mathrm{~cm}^{-1}$ at $200 \mathrm{~K}$. When the initial $2 \mathrm{Cz}-\mathrm{D} /$ EDOT feed ratios were 5:1 and 3:1, the electrical conductivities of the obtained copolymer films were $3.1 \times 10^{-5}$ and $1.5 \times 10^{-4} \mathrm{~S} \mathrm{~cm}^{-1}$ at ambient temperature, respectively. Compared with the P2Cz-D film, little improvements were achieved for the electrical conductivities of the two copolymers. Therefore, the $\sigma-T$ curves of the two copolymers were visually overlapped with the curve of $\mathrm{P} 2 \mathrm{Cz}-\mathrm{D}$ in Figure 7. For the copolymer films obtained with 2Cz-D:EDOT $=1: 1$ and 1:3, their electrical conductivities were 0.01 and $0.04 \mathrm{~S} \mathrm{~cm}^{-1}$ at ambient temperature, and these decreased to 0.003 and $0.01 \mathrm{Scm}^{-1}$ at $200 \mathrm{~K}$, respectively. When the feed ratio decreased to $1: 5$, the obtained copolymer film exhibited a higher electrical conductivity of $0.6 \mathrm{~S} \mathrm{~cm}^{-1}$ at ambient temperature, lower than that of the PEDOT pellet $\left(3.5 \mathrm{~S} \mathrm{~cm}^{-1}\right)$, but four orders of magnitude higher than that of the P2Cz-D film. On the basis of the above discussion, it can be easily deduced that adding more EDOT increased the electrical conductivity of the as-formed copolymer film. With more EDOT added, the increasing electrical conductivities of the copolymers can be explained by the fact that the presence of a sufficient amount of EDOT units in the system greatly completed and enhanced the conjugated structure of the as-formed copolymers. However, when the 2Cz-D/EDOT feed ratio decreased to $1: 10$, the electrical conductivity of the obtained copolymer film was $0.07 \mathrm{~S} \mathrm{~cm}^{-1}$ at ambient temperature, lower than that of the copolymer obtained with feed ratio of 1:5, but the value is still higher than those of other copolymers. This could occur because as increasingly more EDOT was added in the system, the oxidation and polymerization of EDOT became dominant. However, the small amount of $2 \mathrm{Cz}-\mathrm{D}$ in the system would affect the further growth of the conjugated chain of PEDOT because of steric hindrance. As a result, the integrality of the crosslinked structure and the regularity of the conjugated structure were both disturbed, which lowered the electron transfer capability and reduced the electrical conductivity. Moreover, note that each group of data in Figure 7 was repeated with different samples to ensure that the data was accurate and credible.

Figure 8 shows the temperature dependence of the Seebeck coefficients of the PEDOT pellet and some of the obtained copolymer films. 


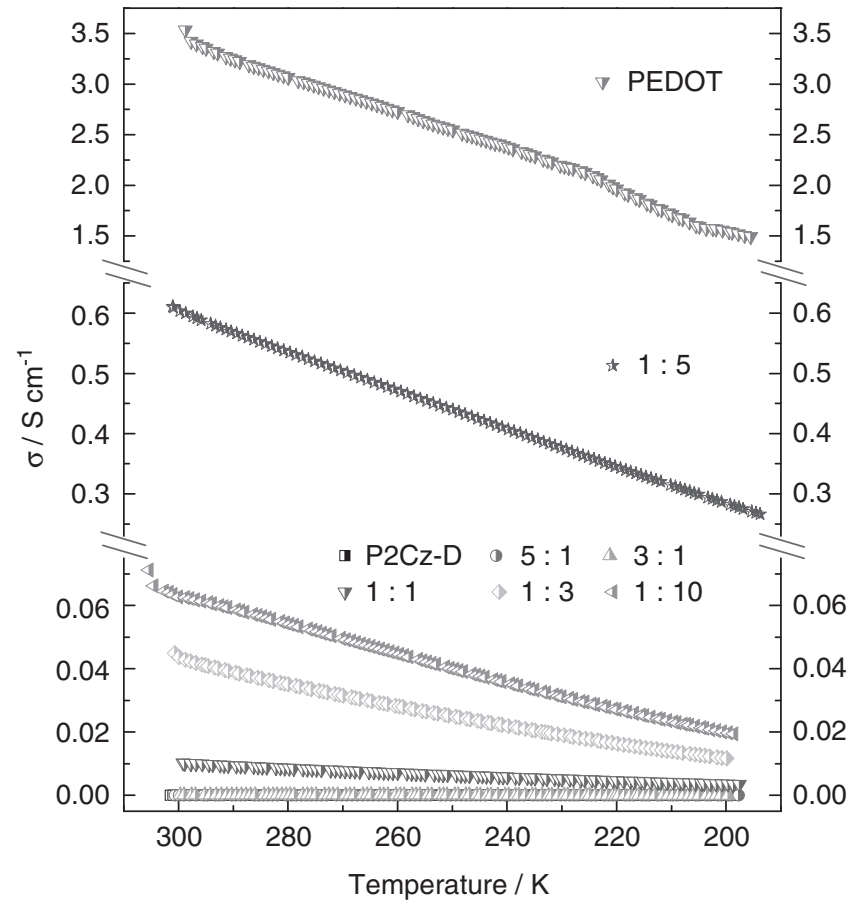

Figure 7 Temperature dependence of the electrical conductivities of $\mathrm{P} 2 \mathrm{Cz}$ D, PEDOT and the copolymers synthesized with 2Cz-D:EDOT=5:1, 3:1, 1:1, 1:3, 1:5 and 1:10 in $\mathrm{CH}_{2} \mathrm{Cl}_{2}+0.1-\mathrm{M} \mathrm{Bu} \mathrm{NBF}_{4}$.

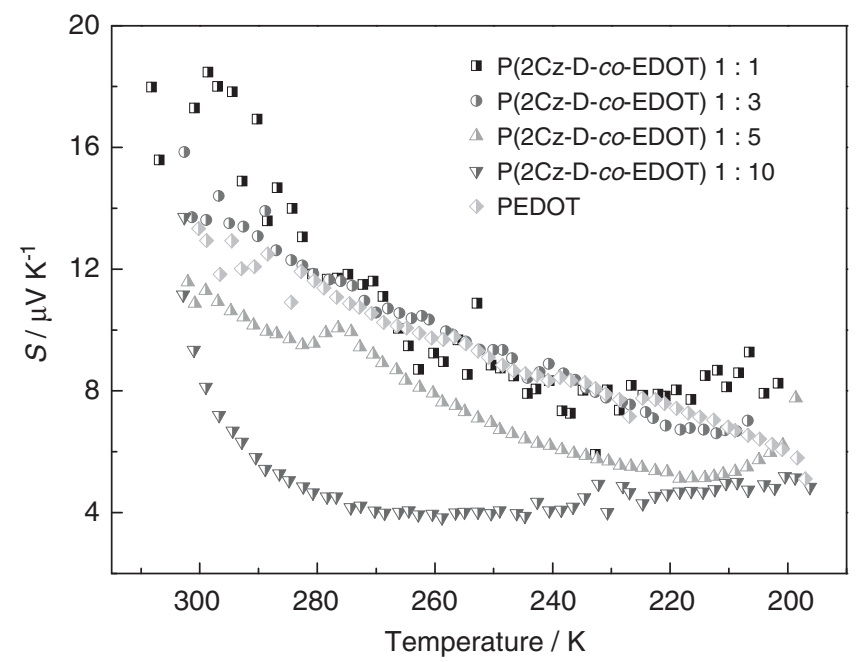

Figure 8 Temperature dependence of the Seebeck coefficients for PEDOT and the copolymers synthesized with 2Cz-D:EDOT=1:1, 1:3, 1:5 and 1:10 in $\mathrm{CH}_{2} \mathrm{Cl}_{2}+0.1-\mathrm{m} \mathrm{Bu} \mathrm{NBF}_{4}$.

The figure shows that as the temperature decreased, most measured Seebeck coefficients showed a downward trend. For PEDOT, the Seebeck coefficient at ambient temperature was $13.3 \mu \mathrm{VK}^{-1}$ and gradually decreased to $5.8 \mu \mathrm{V} \mathrm{K}^{-1}$ at $200 \mathrm{~K}$. After copolymerization, the Seebeck coefficients of the copolymer films obtained with $2 \mathrm{Cz}$ D:EDOT $=1: 1,1: 3,1: 5$ and $1: 10$ were $17.3,13.6,13.3$ and $8.8 \mu \mathrm{V} \mathrm{K}^{-1}$ at $300 \mathrm{~K}$, respectively, comparable to that of PEDOT. For the copolymer obtained with $2 \mathrm{Cz}-\mathrm{D}: \mathrm{EDOT}=1: 10$, the Seebeck coefficient first decreased and then remained almost unchanged as the temperature a
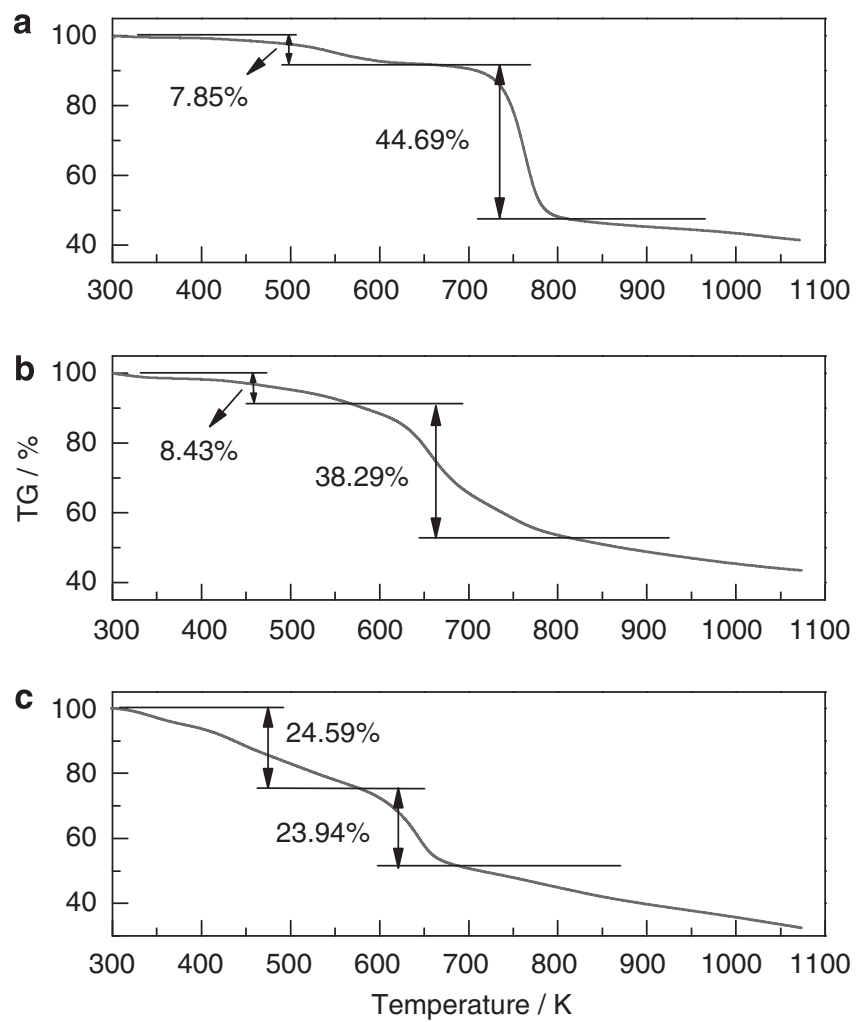

Figure 9 Thermogravimetric curves of dedoped P2Cz-D (a), P(2Cz-D-coEDOT) (2Cz-D:EDOT=1:5) (b) and PEDOT (c) obtained potentiostatically from $\mathrm{CH}_{2} \mathrm{Cl}_{2}+0.1-\mathrm{M} \mathrm{Bu}_{4} \mathrm{NBF}_{4}$.

decreased. However, for P2Cz-D and the copolymer films prepared with 2Cz-D:EDOT=5:1 and 3:1, no Seebeck effect was measured, mainly because of the lower electrical conductivities and limited carrier mobility. Thus, the $S-T$ curves of these materials were not included in Figure 8.

\section{Thermal analysis}

The thermal degradation behavior of CPs is important for their potential applications. For most CPs, their skeletal decomposition temperatures are usually lower than $600 \mathrm{~K}$, which hinder their practical usage in various fields. To investigate the thermal stability of the obtained copolymers, thermogravimetric analytical experiments were performed under nitrogen as shown in Figure 9. This figure depicts three-step weight losses for both the copolymer (Figure 9b) and the homopolymers (Figures 9a and c). Both P2Cz-D and the copolymer (Figures $9 \mathrm{a}$ and $\mathrm{b}$ ) initially underwent small weight losses of $\sim 7.85$ and $8.43 \%$ as the temperature increased from 300 to $676 \mathrm{~K}$ and from 300 to $567 \mathrm{~K}$, respectively, which might be attributed to water evaporation trapped in the polymers. With the gradually increasing temperature, a prominent weight loss of $\sim 44.69 \%$ was found at $676 \mathrm{~K}<T<818 \mathrm{~K}$ for P2Cz-D (Figure 9a), and $\sim 38.29 \%$ at $567 \mathrm{~K}<T<813 \mathrm{~K}$ for the copolymer (Figure 9b), which were both essentially due to the oxidizing decomposition of the skeletal polymer backbone chain structure. The corresponding maximal degradation rate was at $765 \mathrm{~K}$ for $\mathrm{P} 2 \mathrm{Cz}-\mathrm{D}$ and $670 \mathrm{~K}$ for the copolymer. The degradation above 818 or $813 \mathrm{~K}$ was probably caused by the overflow of the oligomers decomposed from the polymer main chains, as mentioned previously. However, the thermogravimetric curve of the copolymer (Figure 9b) shows unclear boundaries between adjacent 
weight-loss steps compared with that of P2Cz-D (Figure 9a). As the temperature increased from 300 to $574 \mathrm{~K}$, the weight of PEDOT gradually decreased (a weight loss of about 24.59\%, Figure 9c). In addition to the reasons outlined above, other explanations may include the loose powder state of PEDOT and the weak C-O bonds in EDOT molecules, which are easily broken at moderate temperatures, making the polymer thermally unstable. About $23.94 \%$ weight loss was observed from 574 to $695 \mathrm{~K}$, indicating the decomposition of skeletal PEDOT backbone chains. When the temperature was higher than $695 \mathrm{~K}$, the decrease in the weight of the polymer still continued because of the continued degradation of the oligomers decomposed from the polymer backbone chain. In addition, when the temperature reached $1000 \mathrm{~K}$, the residual weight of the polymer was $43.81 \%$ for P2Cz-D, 36.83\% for PEDOT and $45.96 \%$ for the copolymer. All the above features demonstrate the high thermal stability of the copolymers. Therefore, this copolymer film can be used over a wide temperature range for potential applications.

\section{CONCLUSION}

$\mathrm{P}$ (2Cz-D-co-EDOT) was electrochemically synthesized by the direct anodic oxidation of $2 \mathrm{Cz}-\mathrm{D}$ and EDOT in $\mathrm{CH}_{2} \mathrm{Cl}_{2}+0.1-\mathrm{M} \mathrm{Bu}_{4} \mathrm{NBF}_{4}$. CVs, FT-IR and morphological and elemental analyses confirm that the resultant polymer is a copolymer with crosslinked structure rather than a composite or a blend of the two homopolymers. As-formed copolymer films show high electrochemical activity and stability, good blue-light emittance, high thermal stability and good mechanical properties. Furthermore, the obtained copolymers are free-standing films with smooth and compact surfaces that can be directly peeled off the electrode surface by hand. The electrical conductivities of the copolymer films were improved compared with that of the P2Cz-D film. The highest electrical conductivity of the copolymer film was $0.6 \mathrm{~S} \mathrm{~cm}^{-1}$, four orders of magnitude higher than that of $\mathrm{P} 2 \mathrm{Cz}-\mathrm{D}$ $\left(2.7 \times 10^{-5} \mathrm{~S} \mathrm{~cm}^{-1}\right)$. In addition, the Seebeck coefficients of the copolymer films were also modified compared with that of the P2Cz-D film. In conclusion, a novel conducting copolymer film that combines the advantages of the homopolymers (P2Cz-D and PEDOT), such as high mechanical properties, good blue-light emittance, high thermal stability and improved thermoelectric properties, has been successfully synthesized and could be applied to photoelectronic fields or used as an organic thermoelectric material.

\section{ACKNOWLEDGEMENTS}

NSFC (50963002\&51073074) and Key Laboratory of Photochemical Conversion and Optoelectronic Materials, TIPC, CAS are acknowledged for their financial supports.

1 Shirakawa, H., Louis, E. J., MacDiarmid, A. G., Chiang, C. K. \& Heeger, A. J. Synthesis of electrically conducting organic polymers: halogen derivatives of polyacetylene, $(\mathrm{CH})_{x}$. J. Chem. Soc. Chem. Commun. 16, 578-580 (1977).

2 Heeger, A. J. Semiconducting and metallic polymers: the fourth generation of polymeric materials. J. Phys. Chem. B 105, 8475-8491 (2001).

3 MacDiarmid, A. G. Synthetic metals: a novel role for organic polymers. Synth. Met. 125, 11-22 (2002).

4 Udum, Y. A., Ergun, Y., Sahin, Y., Pekmez, K. \& Yildiz, A. Electrochemical synthesis and characterization of a new soluble conducting polymer. J. Mater. Sci. 44, 3148-3155 (2009).

5 Winder, C. \& Sariciftci, N. S. Low bandgap polymers for Photon Harvesting in bulk heterojunction solar cells. J. Mater. Chem. 14, 1077-1086 (2004).

6 Kim, Y. G., Thompson, B. C., Ananthakrishnan, N., Padmanaban, G., Ramakrishnan, S. \& Reynolds, J. R. Variable band gap conjugated polymers for optoelectronic and redox applications. J. Mater. Res. 20, 3188-3198 (2005).

7 Reddinger, J. L., Sotzing, G. A. \& Reynolds, J. R. Muticoloured electrochromic polymers derived from easily oxidized bis[2-(3,4-ethylenedioxy)thienyl]carbazoles. Chem. Commun. $15,1777-1778$ (1996)
8 Pickup, D. F., Yi, H. N. \& Iraqi, A. Preparation and properties of alternating 2,7-linked carbazole copolymers with phenylene units with varying number of fluorine substituents. J. Mater. Sci. 44, 3172-3178 (2009).

9 Low, P. J., Paterson, M. A. J., Yufit, D. S., Howart, J. A. K., Cherryman, J. C., Tackley, D. R., Brook, R. \& Brown, B. Towards an understanding of structure-property relationships in hole-transport materials: the influence of molecular conformation on oxidation potential in poly(aryl)amines. J. Mater. Chem. 15, 2304-2315 (2005).

10 MacClenaghan, N. D., Passalacqua, R., Loiseau, F., Campagna, S., Verheyde, B., Hameurlaine, A. \& Dehaen, W. Ruthenium(II) dendrimers containing carbazole-based chromophores as branches. J. Am. Chem. Soc. 125, 5356-5365 (2003).

11 Trannekar, P., Fulghum, T., Baba, A., Patton, D. \& Advincula, R. Quantitative electrochemical and electrochromic behavior of terthiophene and carbazole containing conjugated polymer network film precursors: EC-QCM and EC-SPR. Langmuir 23, 908-917 (2007).

12 Grazulevicius, J. V., Strohriegl, P., Pelichowski, J. \& Pelichowski, K. Carbazole-containing polymers: synthesis, properties and applications. Prog. Polym. Sci. 28, 1297-1353 (2003)

13 Grigoras, M. \& Antonia, N. C. Synthesis and characterization of some carbazole-based imine polymers. Eur. Polym. J. 41, 1079-1089 (2005).

14 Kimoto, A., Cho, J. S., Higuchi, M. \& Yamamoto, K. Synthesis of asymmetrically arranged dendrimers with a carbazole dendron and a phenylazomethine dendron. Macromolecules 37, 5531-5537 (2004).

15 Bloudin, N. \& Leclerc, M. Poly(2,7-carbazole)s: structureproperty relationships. Acc. Chem. Res. 41, 1110-1119 (2008).

16 Watanabe, M., Nishiyama, M., Yamamoto, T. \& Koie, Y. Palladium $/ \mathrm{P}(t-\mathrm{Bu})_{3}$-catalyzed synthesis of $\mathrm{N}$-aryl azoles and application to the synthesis of $4,4^{\prime}, 4^{\prime \prime}$-Tris $(N$-azolyl)triphenylamines. Tetrahedron Lett. 41, 481-483 (2000).

17 Koyuncu, S., Gultekin, B., Zafer, C., Bilgili, H., Can, M., Demic, S., Kay, I. \& Icli, S. Electrochemical and optical properties of biphenyl bridged-dicarbazole oligomer films: electropolymerization and electrochromism. Electrochim. Acta 54, 5694-5702 (2009)

18 Chen, J. P. \& Natansohn, A. Synthesis and characterization of novel carbazolecontaining soluble polyimides. Macromolecules 32, 3171-3177 (1999).

19 Qu, J., Kawasaki, R., Shiotsuki, M., Sonda, F. \& Masuda, T. Synthesis and properties of polyacetylenes carrying $\mathrm{N}$-phenylcarbazole and triphenylamine moieties. Polymer 47 , 6551-6559 (2006).

20 Clouted, E., Olivero, C., Ades, D., Castex, M. C. \& Siove, A. Synthesis and blue luminescence of a soluble newly designed carbazole main-chain polymer. Polymer 43, 3489-3495 (2002).

21 Cosnier, S., Fologea, D., Szunerits, S. \& Marks, R. S. Poly(dicarbazole-N-hydroxysuccinimide) film: a new polymer for the reagentless grafting of enzymes and redox mediators. Electrochem. Commun. 2, 827-831 (2000).

22 Marrec, P., Dano, C., Gueguen-Simonet, N. \& Simonet, J. The anodic oxidation and polymerization of carbazoles and dicarbazoles $\mathrm{N}$-substituted by polyether chains. Synth. Met. 89, 171-179 (1997).

23 Cosnier, S., Lepellec, A., Marks, R. S., Perie, K. \& Lellouche, J. P. A permselective biotinylated polydicarbazole film for the fabrication of amperometric enzyme electrodes. Electrochem. Commun. 5, 973-977 (2003).

24 Diamant, Y., Furmanovich, E., Landau, A., Lellouche, J. P. \& Zaban, A. Electrochemical polymerization and characterization of a functional dicarbazole conducting polymer. Electrochim. Acta 48, 507-512 (2003).

25 Wei, Z. H., Wang, Q., Xu, J. K., Nie, Y. L., Du, Y. K. \& Xia, H. Y. Facile electrosyntheses of high tensile strength alkyl-bridged dicarbazole polymer films and its fluorescence Spectra. J. Polym. Sci. Part A Polym. Chem. 46, 5232-5241 (2008).

26 Jia, P., Xu, J., Ma, J. \& Lu, X. Enhancement of electrochromic contrast by tethering polyaniline onto cyclotriphosphazene. Eur. Polym. J. 45, 772-778 (2009).

$27 \mathrm{Ma}$, L., Li, Y., Yu, X., Yang, Q. \& Noh, C. Fabricating red-blue-switching dual polymer electrochromic devices using room temperature ionic liquid. Sol. Energy Mater. Sol. Cells 93, 564-570 (2009).

28 Ak, M., Şahmetlioğlu, E. \& Toppare, L. Synthesis, characterization and optoelectrochemical properties of poly(1,6-bis(2,5-di(thiophen-2-yl)-1H-pyrrol-1-yl)hexane) and its copolymer with EDOT. J. Electroanal. Chem. 621, 55-61 (2008).

29 Berlin, A., Zotti, G., Zecchin, S., Schiavon, G., Vercelli, B. \& Zanelli, A. New low-gap polymers from 3,4-ethylenedioxythiophene-bis-substituted electron-poor thiophenes. The roles of thiophene, donor-acceptor alternation, and copolymerization in intrinsic Conductivity. Chem. Mater. 16, 3667-3676 (2004).

30 Yildiz, E., Camurlu, P., Tanyeli, C., Akhmedov, I. \& Toppare, L. A soluble conducting polymer of 4-(2,5-di(thiophen-2-yl)-1 H-pyrrol-1-yl)benzenamine and its multichromic copolymer with EDOT. J. Electroanal. Chem. 612, 247-256 (2008).

31 Zhang, C., Hua, C., Wang, G. H., Ouyang, M. \& Ma, C. A. A novel multichromic copolymer via electrochemical copolymerization of (S)-1,1'-binaphthyl-2,2'-diyl bis(N-(6-hexanoic acid-1-yl) pyrrole) and 3,4-ethylenedioxythiophene. Electrochim. Acta 55, 4103-4111 (2010).

32 Wagner, K., Pringle, J. M., Hall, S. B., Forsyth, M., Macfarlene, D. R. \& Officer, D. L. Investigation of the electropolymerisation of EDOT in ionic liquids. Synth. Met. 153, 257-260 (2005).

33 Raimundo, J. M., Blanchard, P., Frere, P., Mercier, N., Ledoux, R. I., Hierle, R. \& Roncali, J. Push-pull chromophores based on 2,2'-bi(3,4-ethylenedioxythiophene) (BEDOT) $\pi$-conjugating spacer. Tetrahedron Lett. 42, 1507-1510 (2001).

34 Walczak, R. M., Cowart, J. S., Abboud, K. A. \& Reynolds, J. R. Conformational locking for band gap control in 3,4-propylenedioxythiophene based electrochromic polymers. Chem. Commun. 15, 1604-1606 (2006). 
$35 \mathrm{Li}$, Y., Ding, J., Day, M., Tao, Y., Lu, J. \& Diorio, M. Synthesis and properties of random and alternating fluorene/carbazole copolymers for use in Blue light-emitting devices. Chem. Mater. 16, 2165-2173 (2004).

36 Li, X. G., Huang, M. R., Duan, W. \& Yang, Y. L. Novel multifunctional polymers from aromatic diamines by oxidative polymerizations. Chem. Rev. 102, 2925-3030 (2002).

37 Otero, T. F. \& Larreta-Azelain, E. D. Electrochemical generation of polythiophene films on platinum electrodes. Polymer 29, 1522-1527 (1988).

38 Morin, J. F., Leclerc, M., Ades, D. \& Siove, A. Polycarbazoles: 25 years of progress. Macromo. Rapid Commun. 26, 761-778 (2005).

39 Innis, P. C., Mazurkiewicz, J., Nguyen, T., Wallace, G. G. \& Macfarlane, D. Enhanced electrochemical stability of polyaniline in ionic liquids. Curr. Appl. Phys. 4, 389-393 (2004).
40 Li, M. C., Ma, C. A., Liu, B. Y. \& Jin, Z. M. A novel electrolyte 1-ethylimidazolium trifluoroacetate used for electropolymerization of aniline. Electrochem. Commun. 7, 209-212 (2005).

41 Kumar, A., Welsh, D. M., Morvant, M. C., Piroux, F., Abboud, K. A. \& Reynolds, J. R. Conducting poly(3,4-alkylenedioxythiophene) derivatives as fast electrochromics with high-contrast ratios. Chem. Mater. 10, 896-902 (1998).

42 Hiroshige, Y., Ookawa, M. \& Toshima, N. High thermoelectric performance of poly(2,5dimethoxyphenylenevinylene) and its derivatives. Synth. Met. 156, 1341-1347 (2006).

43 Lévesque, I., Gao, X., Klug, D. D., Tse, J. S., Ratcliffe, C. I. \& Leclerc, M. Highly soluble poly (2,7-carbazolenevinylene) for thermoelectrical applications: from theory to experiment. React. Funct. Polym. 65, 23-36 (2005).

Supplementary Information accompanies the paper on Polymer Journal website (http://www.nature.com/pj) 